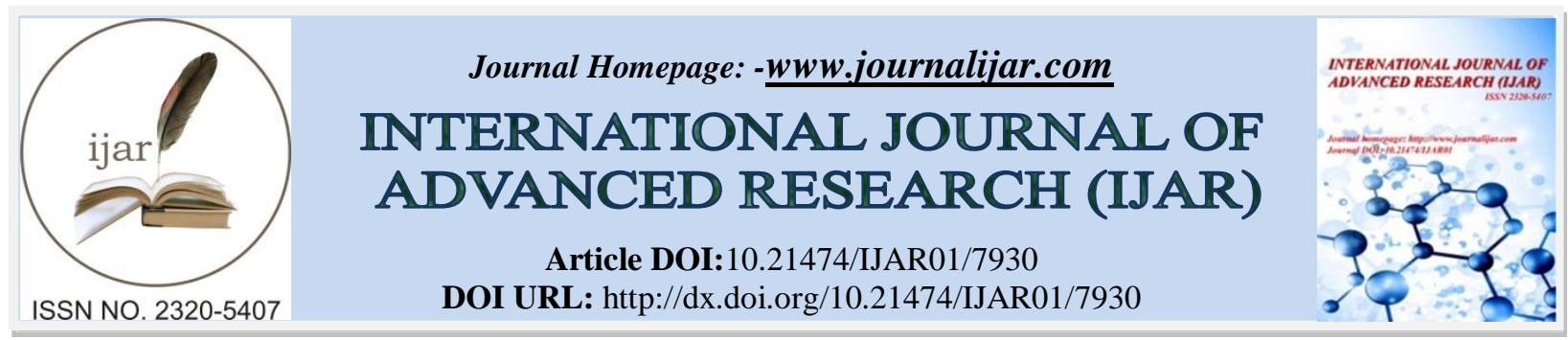

RESEARCH ARTICLE

\title{
CHOLESTEROL MYTH FROM SCRUPULOSITY INTHE 1953 TO CERTITUDE IN 2018.
}

\author{
Abdullah Alabdulagader. \\ Senior Consultant Congenital Cardiologist, Electrophysiologist, Director of Research \&Biostatistics \\ AdministrationWorld Gold Medal Awardee(WOSCO-2012) Prince Sultan Cardiac Centre-Alhassa-Saudi Arabia.
}

\section{Manuscript Info}

Manuscript History

Received: 15 August 2018

Final Accepted: 17 September 2018

Published: October 2018
Abstract

\section{Introduction:-}

Scientific evidence in this historical era is considered in most world communities-if not all- equivalent to holy statement. Angels are the messengers of the holy statements, equivalent in our medical communities to guide lines and policy makers from elite scientific organizations. The stony believe, claiming that cholesterol is harmful to human beings is fallacious .It is great remorse for humanity but interrupting the harm late is better than keeping it for ever. The cholesterol atherosclerosis theory is considered by many authorities in the year 2018 as the greatest myth in medical history. We devoted one day of lectures to discuss the cholesterol theory in our biannual international conference for advanced cardiac sciences (King of Organs Conference Saudi Arabia, 2008) and discussion of this mis believe continued in the next conferences for advanced cardiac sciences (King of Organs 2010-2012). Since then, and even earlier, great wave of international attention around the globe stared to question what was thought to be holy medical believe.Dietary cholesterol claimed to be strongly linked to coronary heart disease in all world nations and across all human races.It has hadenormous impact on health care services, health economics and world population well-being for decades, but has little or no scientific back ground that is relevant for the human species. Indeed, the hypothesis is false and should be buried.1 The historical background of the hypothesis of a causal relationship between the level of serum cholesterol and the development of atherosclerosis began with Rudolf Virchow's (1821-1902) description in 1856 of the atherosclerotic plaque with its cholesterol deposits.2 Nikolai Anichkov's (1885-1964) experiment with rabbits in St Petersburg first demonstrated what thought to be the role of cholesterol in the development of atherosclerosis. He fed rabbits -herbivores- with cholesterol from egg yolks and found that they developed atherosclerotic plaques containing cholesterol. When he tried with other animals - carnivores - it was not possible to reproduce the results. They didn't get atherosclerosis. ${ }^{1}$ In 1915, Anichkov moved to Freiburg to work under Dr. Aschoff, who at that time was considered the most accomplished of all German pathologists. ${ }^{2}$ The scientific reputation of the group in pathology, passed in subtle way to benefit the cholesterol theory.

Food and pharmaceutical industry has its major impact in the development and consolidation of the cholesterol myth. John Gofman, who is physicist and chemist with qualification in medicine, continued the work of Anichove and colleagues. He and his collaborators investigated the body's lipoproteins, which contain both proteins and fats, and their circulation within the bloodstream. They described low-density and high-density lipoproteins and their roles in metabolic disorders and coronary disease. This work continued throughout the late 1940s and early 1950s. Gofman work, apparently attracted attentions of medical communities and was honoured with the title of "Father of Clinical Lipidology" by the Journal of Clinical Lipidology in 2007. ${ }^{3}$ Most of Gofman scientific contribution was in 
the field of radiation-safety science. In Lipidology, He likes all workers in the field before him and after him until the moment, was not able to establish cause effect relationship between cholesterol levels and atherosclerosis.

Two publications by Ancel Keys (1904 - 2004)had a tremendous impact on the general belief of the cholesterol hypothesis. Keys was a Harvard-trained physiologist and epidemiologist. He did some research on human starvation in the mid- $20^{\text {th }}$ century before moving on to study heart disease for which he is probably the most famous. In 1953 he reported that the dietary intake of fat was significantly correlated to the serum cholesterol level and reported theincidence of cardiovascular death in six countries. ${ }^{4}$ It appeared very convincing but the problem was that these six countries were selected from all together 22 countries. In 1958 he launched the Seven Countries Study, after exploratory research on the relationship between dietary pattern and the prevalence of coronary heart disease in Greece, Italy, Spain, South Africa, Japan, and Finland. There was no correlation whatsoever if all the countries were included. The study was obviously a falsification. With statistical manoeuvres he "showed" that saturated fat was the culprit. ${ }^{1}$ Keys mentionedthat only a few countries are available for any kind of real comparison at the time of his publication. Some countries he leaves out of the graph because of major population shifts or poorlymaintained vital health statistics. He does mention that there are good quality health statistics available for many other European countries, but since World War 2 had such an effect on diets, the food data of Nazi-occupied territories were left out. What is left appears to be a remarkable relationship between fat intake and death.

The other publications came in year 1986, 15 cohorts of the Seven Countries Study, comprising 11,579 men aged 40 -years and "healthy" at entry, 2,288 died in 15 years. ${ }^{5}$ At this time, the conclusion of Keys et al came with flavour of remorse: All- cause and coronary heart disease death rates were low in cohorts with olive oil. The main fat causal relationships are not claimed but consideration of characteristics of populations as well as of individuals within population is urged evaluating risks. Keys' publication is an ethical and tragic turning point in the history of the field. It is Keys publication and the forces behind it that create the anti-cholesterol agents and in particular statin dilemma in the medical communities. The propagation and the unprecedented enthusiasm to his conclusions were abused by the pharmaceutical and food industries since 1953 until the moment. ${ }^{2}$

Jacob Yerushalmy and Herman E. Hilleboe pointed out that, for an earlier study demonstrating this association, Keys had selected six countries out of 22 for which data were available. Analysis of the full dataset made the analysis between fat intake and heart disease less clear. In 1957 when they published their critique, Yerushalmy and Hilleboe stated: "the association between the percentage of fat calories... and mortality from... heart disease is not valid" and then they call Keys's work a "tenuous association". ${ }^{6}$

A critical assessment of research on the reduction of cholesterol levels by statin treatment to reduce cardiovascular disease was published recently. ${ }^{7}$ Although, statins are effective at reducing cholesterol levels; they have failed to substantially improve cardiovascular outcomes. The deceptive approach statin advocates have deployed to create the appearance that cholesterol reduction results in an impressive reduction in cardiovascular disease outcomes was described.

This historical deception was through their use of a statistical tool called relative risk reduction (RRR), a method which amplifies the trivial beneficial effects of statins. David Diamond and UffeRavnskov in this prestigious publication described how the directors of the clinical trials have succeeded in minimizing the significance of the numerous adverse effects of statin treatment. ${ }^{7} \quad$ We and others have raised major questions in this direction and acknowledged that statin treatment has been shown to reduce mildly and non-impressively coronary events. ${ }^{8,9}$ Close inspection reveals that the benefit is much less impressive than clinicians and the general public have been told and that it must be because of other mechanisms than cholesterol reduction. ${ }^{10,11,12}$

In another prestigious publication in BMJ UffeRavnskov and elite group of colleagues published a systematic review on the Lack of an association or an inverse association between low-density lipoprotein cholesterol and mortality in the elderly, ${ }^{13}$ considering its scientific content and the prestigious names of co-authors this review is a landmark in the field. In the studies reviewed an explanation for the increased risk of mortality among people with low cholesterol is that serious diseases may lower cholesterol soon before death occurs. Evidence to support this hypothesis may be obtained from 10 of the studies in which no exclusions were made for individuals with terminal illnesses. However, in four of the studies, participants with a terminal illness or who had died during the first observation year were excluded. In one of those studies,LDL-C was not associated with all-cause mortality. ${ }^{14}$ While in the three others, Akerblom JL et al; concluded that Whites and African-Americans in the lowest quartiles 
of total cholesterol, non-HDL cholesterol and LDL cholesterol were approximately twice as likely to die as those in the highest quartile, in contrast plasma lipid levels were not related to mortality risk among Hispanics. ${ }^{15}$ On the other hand, Bathum L, et al; stated that these associations indicate that high lipoprotein levels do not seem to be definitely harmful in the general population. However, high triglyceride levels in females are associated with decreased survival. ${ }^{16}$ Nevertheless, LV YB et al; concluded that among the Chinese oldest old, higher LDL-C level was associated with lower risk of all-cause mortality. ${ }^{17}$

In UffeRavnskov et $\mathrm{al}^{10}$ review, LDL-C was inversely associated with all-cause mortality and with statistical significance. Thus, there is a little support for the hypothesis that the authors' analysis is biased by end of life changes in LDL-C levels.

After publication of this study, the international medical community was expecting massive reactions from lipid hypothesis and cholesterol advocates. Nunan et $\mathrm{al}^{18}$ from the Centre of Evidence Based Medicine (CEBM), University of Oxford statement was the most important barely reasonable response. It is serious disappointment to turn the scientific discussion to a true deviation. The truth is most difficult to hide. The whole statement is a weak continuation to the past trials of selective creation of correlation in spite of our crystal clear challenge to confirm causation. It is painful for us as international medical community to read the statement criticism on UffeRavnskov and colleagues' review. ${ }^{2}$ UffeRavnskov responded to the statement "It is very wise to ask Nunan et al to show us a study where the authors have found the opposite." What they have shown is that lower genetically determined LDL cholesterol concentrations are associated with lower all-cause mortality, but association does not mean causation. Other genes in the same individual may have opposite effects. ${ }^{2}$

In The British Heart Protection Study of 20536 UK adults (aged 40-80 years) with coronary disease, other occlusive arterial disease, or diabetes were randomly allocated to receive $40 \mathrm{mg}$ simvastatin daily (average compliance: $85 \%$ ) or matching placebo (average non-study statin use: 17\%). Analysis of the first occurrence of particular events, compared all simvastatin-allocated versus all placebo-allocated participants. The authors' final statement in their results interpretation was as follows: The size of the 5-year benefit depends chiefly on such individuals' overall risk of major vascular events, rather than on their blood lipid concentrations alone. ${ }^{19}$

The Anglo-Scandinavian Cardiac Outcomes Trial-Lipid Lowering Arm (ASCOT-LLA) ${ }^{20}$ is considered by lipid hypothesis advocates as one of the major pillars in the field. This trial included 10,305 individuals with hypertension. In addition, all of them had at least three of the following risk factors: Type 2 diabetes, left ventricular hypertrophy, peripheral arterial disease, previous stroke or transient ischemic attack, or smoking. Half of them received $10 \mathrm{mg}$ atorvastatin, half of them a placebo and the primary endpoint was nonfatal and fatal CHD. The trial was planned to continue for 5 years, but the authors found the preliminary findings so impressive that the study was terminated at 3.3 years. The reason was that at that time 'cholesterol lowering with atorvastatin $10 \mathrm{mg}$ conferred a $36 \%$ reduction in fatal CHD and nonfatal MI compared with placebo'. However, the benefit was actually unimpressive. In the placebo group, $3 \%$ suffered heart attack vs. $1.9 \%$ in the atorvastatin group. Thus, the absolute risk reduction (ARR) was only 1.1 percentage points, which is $36 \%$ of 3 . Moreover, there was no significant benefit in subgroups of patients at high risk of CHD, including those with diabetes, left-ventricular hypertrophy and previous vascular disease or for patients aged 60 years or younger, for those without renal dysfunction and for individuals with metabolic syndrome. For women there were no benefits at all. Indeed, there was a trend for worse, albeit non-significant, effects. Finally, there was no effect on either cardiovascular or non-cardiovascular mortality. ${ }^{7}$

Michel de Lorgeril and Mikael Rabaeus, ${ }^{21}$ in their unique publication examined the reality of safety and efficacy of statins as stated by company-sponsored reports.

They emphasise on a very important fact in the field of cholesterol theory and its contribution to atherosclerosis. This is the introduction of new stricter regulations in the conduct and publication of randomized controlled trials (RCTs).

This would imply that RCTs were less reliable before 2006 than they were later on. To evaluate this, they reviewed RCTs testing the efficacy of statins versus placebo in preventing cardiovascular complications and published after2006. 
Their systematic review identified four major RCTs, all testing rosuvastatin. They unambiguously showed that rosuvastatin is not effective in secondary prevention, while the results are highly debatable in primary prevention.

They then examined the most recent RCTs comparing statins to each other: all showed that no statin is more effective than any other, including rosuvastatin. Furthermore, they showed recent RCTs clearly indicate that intense cholesterol-lowering (including those with statins) does not protect high-risk patients any better than less-intense statin regimens. In addition they documented inefficacy of statins in chronic heart failure and chronic kidney failure patients as well as absence of protection to diabetic patients. They concluded that their review strongly suggests that statins are not effective for cardiovascular prevention. The studies published before 2005/2006 was probably flawed, and this concerned in particular the safety issue. A complete reassessment is mandatory. Until then, physicians should be aware that the present claims about the efficacy and safety of statins are not evidence based. ${ }^{21}$

Recently, celluar biochemistry research creates another massive strike to the lipid hypothesis. In recent publication in the journal of cellular biochemistry by ShuanShian Huang and colleagues, ${ }^{22}$ opened new era of respectful scientific understanding about the true mechanism of the plaque formation. The authors were activated by the new epidemiological evidence documenting absence of support of the claimed relationship between dietary cholesterol and/or blood cholesterol and atherosclerosis. These results suggest that 7-dehydrocholesterol (7-DHC; the biosynthetic precursor of cholesterol), but not cholesterol, promotes lipid raft/caveolae formation, leading to suppression of canonical TGF- $\beta$ signalling and at atherogenesis.

Statin advocates spent decades claiming that circulating biomarkers of oxidized low-density lipoprotein (OxLDL) are affected by statin therapy and predict changes in atheroma volume. SeungHyuk Choi and colleagues, ${ }^{23}$ in a unique study published in the American Journal of Cardiology -Observations From the REVERSAL (Reversal of Atherosclerosis with Aggressive Lipid Lowering) Study-examined the Relationship Between Biomarkers of Oxidized Low-Density Lipoprotein, Statin Therapy, Quantitative Coronary Angiography, and Atheroma Volume. They concluded that, Statin therapy results in significant increases in OxPL/apoB, MDA/apoB, and lipoprotein (a) levels and decreases in apoB immune complexes and OxLDL auto-antibodies. However, these measures did not correlate with changes in Quantitative coronary angiography QCA parameters or atheroma volume.

In the near future we are expecting what seems to be groundbreaking news in the time line of the history of cholesterol myth in the Novartis Phase III CANTOS study targeting the subtle inflammatory bases theory of atherosclerosis. Its initial results demonstrated that targeting inflammation with ACZ885 reduces cardiovascular risk. ACZ885 (canakinumab) is a selective, high-affinity, fully human monoclonal antibody that inhibits IL-1ß, a key cytokine in the inflammatory pathway known to drive the continued progression of inflammatory atherosclerosis. ACZ885 works by blocking the action of IL-1ß for a sustained period of time, therefore inhibiting inflammation that is caused by its over-production.

The study early results showed that ACZ885 led to a statistically significant $15 \%$ reduction in the risk of major adverse cardiovascular events (MACE), a composite of non-fatal heart attack, non-fatal stroke and cardiovascular death, compared to placebo ( $\mathrm{p}$-value 0.021).This apparently impressive outcome occurred without touching cholesterol levels. It is very critical for CANTOS investigators as part of their historical responsibility in front of the word communities not to express deformed statistics from this major trial. All results MUST BE expressed by absolute risk reduction (ARR) rather than relative risk reduction (RRR) which falsely exaggerate the findings.

In contrast to the current belief that cholesterol reduction with statins decreases atherosclerosis, Okuyama $\mathrm{H}$ and colleagues, ${ }^{24}$ presented a perspective that statins may be causative in coronary artery calcification and can function as mitochondrial toxins that impair muscle function in the heart and blood vessels through the depletion of coenzyme Q10 and 'heme A', and thereby ATP generation. Statins inhibit the synthesis of vitamin K2, the cofactor for matrix Gla-protein activation, which in turn protects arteries from calcification. Statins inhibit the biosynthesis of selenium containing proteins, one of which is glutathione peroxidase serving to suppress peroxidative stress. An impairment of selenoprotein biosynthesis may be a factor in congestive heart failure, reminiscent of the dilated cardiomyopathies seen with selenium deficiency. Thus, the epidemic of heart failure and atherosclerosis that plagues the modern world may paradoxically be aggravated by the pervasive use of statin drugs. They propose that current statin treatment guidelines must_be critically re-evaluated. 
In a recent publication the main risk factors for atherosclerosis patients were examined by Wei Luo and colleagues,

25 they concluded: Hyperlipidemia is not an important cause of coronary atherosclerosis. High glucose levels and glycosylated lipoprotein are of high importance in the development and progression of coronary atherosclerosis.

We have been overwhelmed in the whole spectrum of medical communities in the last few decades with the idea of lipoprotein retention model of atherosclerosis. This model of atherosclerosis formation stated that: The key initiating process in atherogenesis is the subendothelial retention of apolipoprotein B-containing lipoproteins. Local biological responses to these retained lipoproteins, including a chronic and maladaptive macrophage and $\mathrm{T}$-celldominated inflammatory response, promote subsequent lesion development. ${ }^{25}$ This theoretical model was considered as fact based in the -claimed- unequivocal success of LDL-lowering therapy to treat atherosclerosis. It is unfortunate, to announce to all medical communities that this, claimed, unequivocal success was, merely, statistical deception.

The truth is always derived from the scrutinized unbiased and rigorous epidemiological studies, in contrast to cherry picking and deceptive data management studies.Epidemiological studies are known to have the major directive force towards disclosing scientific mysteries compared to the assumed theories.Cholestrol myth is prominent example in this direction.All-cause mortality is the most appropriate outcome to use when investigating risk factors for life threatening disease. Large epidemiological studies in Japan showed clearly overall, an inverse trend between allcause mortality and total (or low density lipoprotein [LDL]) cholesterol levels: mortality is highest in the lowest cholesterol group without exception. If limited to elderly people, this trend is universal. Elderly people with the highest cholesterol levels have the highest survival rates irrespective of where they live in the world. ${ }^{26}$ The largest epidemiological study the Ibaraki Prefecture Health Study carried out in Japan in recent years. Men and women $(\mathrm{n}=$ 91,219) aged 40-79 years with no history of stroke or coronary heart disease (CHD) were followed for 10.3 years. The hazard ratio (HR) of all cause mortality adjusted for age and many potential confounding factors was calculated according to LDL cholesterol levels and revealed that all-cause mortality was essentially inversely correlated with LDL cholesterol levels in both men and women. ${ }^{27}$

This is the level of evidence which must be presented to physicians worldwide in contrast to the deceptive manipulated data overwhelming our medical communities.

In the present time, the massive flow of data is continuously accumulating about the drawback and side effects of statins. Almost all vital systems and organs are targeted by statin medications. Increasing number of epidemics of myopathy is universally well known side effect but other major human disasters are rising up secondary to statin therapy. Depressed lipoproteins and its tragic reflections in immune status especially in elderly as well as cancer, diabetes millets , loss of memory, lower cognitive functioning, dementia, depression ,suicidal behaviour, and many others, are alarming hazards to humanity in the late half of 2018.

This review is not intended to list all statins side effects but those tragic side effects mandate serious ethical questioning for immediate guide lines review to save human kind from a medical deception that should come to an end.The level of evidence is more than trials to hide it.

It is time for scrupulosity of cholesterol theory created in 1953 to be replaced in the year 2018 with certitude.

\section{References:-}

1. Scherstén T, Rosch PJ, Arfors KE, Sundberg R. The cholesterol hypothesis: time for the obituary? ScandCardiovasc J. 2011 Dec;45(6):322-3.

2. Alabdulgader Abdullah, Global Consciousness and The Great Cholesterol Myth (from1800s to 2018): Wisdom Must Prevail. Review paper under press.

3. Gofman JW, Young W, Tandy R. Ischemic heart disease, atherosclerosis, and longevity. Circulation. 1966 Oct;34(4):679-97.

4. KEYS A. Atherosclerosis: a problem in newer public health. J Mt Sinai Hosp NY. 1953 Jul-Aug;20(2):118-39.

5. Keys A, Menotti A, Karvonen MJ, Aravanis C, Blackburn H, Buzina R, Djordjevic BS, Dontas AS, Fidanza F, Keys $\mathrm{MH}$, et al. The diet and 15-year death rate in the seven countries study. Am J Epidemiol. 1986 Dec;124(6):903-15.

6. Yersushalmay J, HilleboeHE.Fat in the diet and mortality from heart disease;amethodologic note. N Y State J Med. 1957 Jul 15;57(14):2343-54.

7. Diamond DM, Ravnskov U. How statistical deception created the appearance that statins are safe and effective in primary and secondary prevention of cardiovascular disease. Expert Rev ClinPharmacol. 2015 Mar;8(2):201-10. 
8. Alabdulgader Abdullah; The Major Chloestrol Myth: ,Proceedings of King of Organs Interrnational Conferences for Advanced Cardiac Sciences (2006,2008,1010,2012), ISBN 978-9952-451-29-0, World Organization for Scientific Cooperation (WOSCO) Part 2,Munich ,Germany, 2017.

9. Alabdulgader Abdullah (2016) Future of cardiovascular practice: Alert to change or call for revolution.. J Cardiovasc Med Ther. 2017;1(1):1.

10. Ravnskov U. A hypothesis out-of-date. the diet-heart idea. J Clin Epidemiol.2002 Nov;55(11):1057-63.

11. Lindholm LH, Samuelsson O; ASCOT. What are the odds at ASCOT today? Lancet.2003 Apr 5;361(9364):1144-5.

12. Thompson A, Temple NJ. The case for statins: has it really been made? J R Soc Med. 2004 Oct;97(10):461-4.

13. Ravnskov U, Diamond DM, Hama R, Hamazaki T, Hammarskjöld B, Hynes N, Kendrick M, Langsjoen PH, Malhotra A, Mascitelli L, McCully KS, Ogushi Y, Okuyama H, Rosch PJ, Schersten T, Sultan S, Sundberg R. Lack of an association or an inverse association between low-density-lipoprotein cholesterol and mortality in the elderly: a systematic review. BMJ Open. 2016 Jun 12;6(6):e010401.

14. Zimetbaum P, Frishman WH, Ooi WL, Derman MP, Aronson M, Gidez LI, Eder HA.Plasma lipids and lipoproteins and the incidence of cardiovascular disease in the very elderly. The Bronx Aging Study. ArteriosclerThromb. 1992 Apr;12(4):416-23.

15. Akerblom JL, Costa R, Luchsinger JA, Manly JJ, Tang MX, Lee JH, Mayeux R, Schupf N. Relation of plasma lipids to all-cause mortality in Caucasian, African-American and Hispanic elders. Age Ageing. 2008 Mar;37(2):207-13.

16. Bathum L, Depont Christensen R, Engers Pedersen L, Lyngsie Pedersen P, Larsen J, Nexøe J. Association of lipoprotein levels with mortality in subjects aged $50+$ without previous diabetes or cardiovascular disease: a population-base register study. Scand J Prim Health Care. 2013 Sep; 31(3):172-80.

17. Lv YB, Yin ZX, Chei CL, Qian HZ, Kraus VB, Zhang J, Brasher MS, Shi XM, Matchar DB, Zeng Y. Low-density lipoprotein cholesterol was inversely associated with 3-year all-cause mortality among Chinese oldest old: data from the Chinese Longitudinal Healthy Longevity Survey. Atherosclerosis. 2015 Mar;239(1):137-42.

18. David Nunan, Dylan Collins, Nikolas Bobrovitz, Kamal R. Mahtani. CEBM response: "Lack of an association or an inverse association between low-density-lipoprotein cholesterol and mortality in the elderly: a systematic review" - a post publication peer review. June 13, 2016

19. https://www.cebm.net/2016/06/cebm-response-lack-association-inverse-association-low-density-lipoproteincholesterol-mortality-elderly-systematic-review-post-publication-pee/. Accessed on 25/09/2018.

20. Heart Protection Study Collaborative Group. MRC/BHF Heart Protection Study of cholesterol lowering with simvastatin in 20,536 high-risk individuals: a randomised placebo-controlled trial.Lancet. 2002 Jul 6;360(9326):7-22.

21. Sever PS, Dahlöf B, Poulter NR, Wedel H, Beevers G, Caulfield M, Collins R, Kjeldsen SE, Kristinsson A, McInnes GT, Mehlsen J, Nieminen M, O'Brien E, Ostergren J; ASCOT Investigators. Prevention of coronary and stroke events with atorvastatin in hypertensive patients who have average or lower-than-average cholesterol concentrations, in the Anglo-Scandinavian Cardiac Outcomes Trial--Lipid Lowering Arm (ASCOT-LLA): a multicentrerandomised controlled trial. Drugs. 2004;64Suppl 2:43-60.

22. deLorgeril M, Rabaeus M. Beyond Confusion and Controversy, Can We Evaluate the Real Efficacy and Safety of Cholesterol-Lowering with Statins?. Journal of Controversies in Biomedical Research 2015;1(1)

23. Huang SS, Liu IH, Chen CL, Chang JM, Johnson FE, Huang JS. 7-Dehydrocholesterol (7-DHC), But Not Cholesterol, Causes Suppression of Canonical TGF- $\beta$ Signaling and Is Likely Involved in the Development of Atherosclerotic Cardiovascular Disease (ASCVD). J Cell Biochem. 2017 Jun;118(6):1387-1400.

24. Choi SH, Chae A, Miller E, Messig M, Ntanios F, DeMaria AN, Nissen SE, Witztum JL, Tsimikas S. Relationship between biomarkers of oxidized low-density lipoprotein, statin therapy, quantitative coronary angiography, and atheroma:volume observations from the REVERSAL (Reversal of Atherosclerosis with Aggressive Lipid Lowering) study. J Am CollCardiol. 2008 Jul 1;52(1):24-32.

25. Okuyama H, Langsjoen PH, Hamazaki T, Ogushi Y, Hama R, Kobayashi T, Uchino H. Statins stimulate atherosclerosis and heart failure: pharmacological mechanisms. Expert Rev ClinPharmacol. 2015 Mar;8(2):189-99.

26. Luo W, He Y, Ding F, Nie X, Li XL, Song HL, Li GX. Study on the levels of glycosylated lipoprotein in patients with coronary artery atherosclerosis. J Clin Lab Anal. 2018 Aug 12:e22650.

27. Tabas I, Williams KJ, Borén J. Subendothelial lipoprotein retention as the initiating process in atherosclerosis: update and therapeutic implications. Circulation. 2007 Oct 16;116(16):1832-44.

28. Hamazaki T, Okuyama H, Ogushi Y, Hama R. Towards a Paradigm Shift in Cholesterol Treatment. A Reexamination of the Cholesterol Issue in Japan. Ann NutrMetab. 2015;66Suppl 4:1-116. 